\title{
Intension and Extension Study about Modern Machine Interface Design
}

\author{
Chen $\mathrm{Xu}$ \\ School of Art and Design, Guilin University of Electronic \\ science and Technology, Guilin 541004, China \\ chenxu1968@gmail.com \\ *Chen xu
}

\begin{abstract}
HMI" means the area of mutual influence between the human-machine, who participate in all areas of human-computer exchange of information are part of the man-machine interface. The design is the study of human art, not a simple machine and equipment subject, object by object relations representative, but there is the breadth and depth of things; the people here are not "living beings", not simply to human physiology features were analyzed. Human scale, both should be as natural scale, there should be a social human scale; both studies the physical, psychological and environmental impact on people and performance, but also the study of human culture, aesthetics, values and other requirements and change.
\end{abstract}

Keywords-Human-Machine Interface; Art and Design; Human - Computer Interaction; Design and Analysis

\section{INTRODUCTION}

Interface design and objects exist in the exchange of information, and even can be said that the exchange of information in all areas of existence and objects belong to the design surface, its content elements is extremely broad. Can be defined as the design interface design in the face, the sum of all of the analyzed information exchange, it reflects the relationship between persons and things.

\section{DESIGN OF INTERFACE IN THE PRESENCE OF DIVERSITY}

Design of artificial objects is to grasp the internal and external environment joint discipline, this joint is carried out on people. "People" is a design interface, is the object of the subject of cognition and design service, and as the object of "object" is another aspect of the design interface. It contains complex object entity, environment and information, such as a product, a building, it brings people not only use function, the quality of materials, but also contains the traditional thinking, cultural reason, such as the concept of cognitive science. Any piece of content, must be beyond the individual object representation contained in the works. Analysis of "matter" is also analyzed the diversity of existing design interface.

\section{A Functional design interface}

The function of information received, manipulation and control, also included with the production interface, namely the use of materials, science and technology application etc.. This reflects the coordination effect of interface design and artifacts.

B Emotional design interface
To transfer the feelings of people, and people have feelings of sympathy. This feeling of message exists in the unity of certainty and uncertainty. Emotion is deeply grasp the target object of the user's feelings, not personal emotions, to avoid any subjective and personal free play of individuality. This interface reflects the relationship between design and human.

\section{$C$ Environmental design interface}

The external environmental factors of human information transmission. Any one product could not exist without its environment, physical environmental conditions and spiritual atmosphere interface factors indispensable. Interface design is based on functional interface based on environment, interface as the premise, affective interface as the center of gravity and form, the formation of organic and systematic relation between them.

\section{THE METHODOLOGICAL SIGNIFICANCE TO DESIGN THE INTERFACE EXISTS}

In the era of knowledge economy at present, in meeting the material needs of the situation, the development of people the pursuit of their own personality and emotional appeal, the design must be focus on the people's emotional needs to consider. Design of complex factors lead to the difficulty of design evaluation standard. Analysis using the interface, it is to make the design factors structured, avoid the subjective one sidedness.

\section{A Function is not single, it includes the use of function, aesthetic function, social function, environmental function etc..}

Excessive pursuit of single function will lead to many of the most important contents (adornment sex, nationality, intermediate) be rejected. And the concept of some content is not function can include, besides the substance and spirit of the content is not always the same amount and quality of harmony in a unity, with the different products, different times, their primary and secondary status changes. In today's information technology high development period, the emotion factor has increasingly become the main aspects of the design. The material sense of function in maintaining the basic status of the case, is not representative of the emotional appeal of the expression;

\section{$B$ While the "form follows function" products, for not}

familiar with its users is difficult to understand.

Products to be for people to understand, the letter must be using a recognized code that symbol system; 
C Meet the same features of the product is not the only form already.

The social and economic development to a certain extent, can appear the demand for professional design, while the people's basic material needs have been able to meet, simply to the material function to determine the design is not appropriate. On the contrary, interface design reflects the essence of human beings and objects to exchange information, but also the design of art connotation, which includes design aspects, and make clear the goal and the program design.

\section{CONNOTATION AND DENOTATION ANALYSIS OF DESIGN INTERFACE}

Classification of the design interface is not be completely absolute, interacts with the overlap between may interface, such as religious culture is a kind of environmental factors, but it brings to the believers often is more religious emotion factors. Here the environment and emotion is not easy to distinguish, but this does not prevent different classification between the existence of substantial differences.

\section{A Analysis of functional interface}

The functional interface, it is the realization of the use of any content, a product of its existence value lies in the use of the first is involved, by the use of two and material analysis of various functional factors and technical method of realizing the function application. On the one hand, and the analysis of the existing thinking is a kind of rational thinking. If the treatment as a way to design the product, then the product will make a variety of features (such as nationality, pure neutral) factors, if the removal of trademark products, it is difficult to recognize is which country or which company products. Of course, this also shows that the existence of a common factor of product, it makes the whole mankind can react in the same way. Feeling and the judgment ability has a common characteristic of objectivity, the international.

Functional interface design to establish based on semiology. The functional design of the interface, inevitably have to let users understand the function operation. Each operation of the people should be in accordance with the logic, is the human nature, and on the mechanical, electronic it should be accurate, no doubt, that the information transfer is the core connotation of function interface.

\section{$B$ Analysis of affective interface}

Any one product or work only with human emotions resonate can be accepted by people, also reflects the charm of design works. The development of modern semiotics is increasingly open in this field, in an effort to make this uncertainty to be compressed, strengthen the rational composition.

Semiotics is also used for the analysis using the human senses and communication, music, dance, clothing, decoration and so on as the symbol system to be analyzed, which are designed for art provides valuable reference and technical means and emotional interface design method of value.

\section{$C$ Analysis of the environmental interface}

Factors covered environment interface design is very wide, it includes political, historical, economic, cultural, science and technology, national, interface design, this reflects the social nature of design art. Characteristic factors of design art of the interface exists and means for understanding clear, reasonable method, there are rules, based on the rational and irrational. Successful works are the perfect deal with these three interfaces of crystallization. As Ieoh Ming Pei designed the Le Louvre Museum expansion project, functional process very well, not to succumb to the damage form and function; but at the same time, through the new material and form reflect the times characteristic and aesthetic tendency of new, this is the environment model of the interface; people watching Le Louvre Museum, not to the ancient times, but with the new values to re - examine, appreciate, triangular appearance it accords with people's psychological expectations, this is the emotion of the interface in the extreme.

\section{APPLICATION OF THE PRINCIPLE OF DESIGN INTERFACE}

\section{A The principle of rationality}

That is, to ensure the system design is based on the reasonable and clear. The analysis of any design should have both qualitative and quantitative, rational and perceptual thinking combined. Efforts to reduce non rational factors, and to improve the quantitative optimization, based. Design should not repeat word for word what others say, must be in the right, the system on the basis of facts and data, carries on the strict theoretical analysis, to persuade through reasoning, with emotion.

\section{$B$ The dynamic principle}

That is, there must be a four-dimensional space or five dimensional space operation concept. A piece of work is not only a two-dimensional plane or ruin stereo, also want to have the transformation of time and space, emotion and thinking and cognition as well as the evolution of multidimensional factors.

\section{$C$ The principle of diversification}

Namely the design varied factors to consider. The current design information is more and more abundant, how to acquire effective information, how to design and analysis of information, is a creative thinking and method of process system.

\section{Interactive principle}

That interface design emphasizes the interactive process. Hand is to convey information, on the other hand is to accept and feedback of people, of any material information to understand and grasp the initiative.

\section{E General principles}


Harmonization is to grasp the three class interface, function, emotion, environment is not isolated and there.

\section{SUMMARY}

Design factors of interface contains is very extensive, but in the use of only emphasis, there is emphasis on hold. Although many design factors, but it is still an integral whole. It is the result of a materialized form, but this form is representative of the era, ethnic and other aspects of consciousness, and ultimately reflect people's "beauty" of the psychological activity.

The use of the design interface, the core is design analysis. Design of mobile phone such as Apple Corp, the first not to draw the "beauty" of the shape and taking account of technical progress, but the user's daily behavior analysis, analysis of his family, likes or dislikes, analysis of his daily behavior, and then investigate the use of mobile phone and in what situations, thus provides the concepts and objectives for the design, designers have the concept and the goal is clear, and with the information interaction produce creativity.

On the other hand, the designers themselves should recognize and study in-depth on the social environment, have a clear understanding of the design work orientation. Therefore, in order to design the interface, rational knowledge is the first, the second is the creative, but also there is analysis, the effectiveness of information processing. Design is not immutable and frozen, the analysis method is not immutable and frozen, interface design is also changing the exchange of information on the people and things in the development.

\section{ACKNOWLEDGEMENT}

This research was financially supported by the Guangxi philosophy and social science project"Zhuang brocade technique productive protection and Northern Tourism Development Strategies of cultural and creative industries" (13BXW008)

\section{REFERENCES}

[1] Chen xu. Analysis of the psychology factors in the product form design[J]. Machine Design, 2013, 281, 105-106.

[2] Chen xu. Practice and thinking of the reform in education of art and design[C]//Proceedings of the 2008 International Conference on Industrial Design, 2008: 372-375.

[3] B Joseph Pyne and James H Gilmore. The experience economy [M]. Machinery Industry Press, 2002.

[4] Chen xu. Expression of Design Implication for the Products in the Digital Environment[C]//2009 International Symposium on Computational Intelligence and Design, 2009:153-157。

[5] Mark - Janine, Editor, Non material society. Sichuan people's Publishing House Publishers.(2005)

[6] Zhu Xiaoyue,Editor, Discussion on art design. Jiangxi Fine Arts Publishing House.(2002)

[7] Information on http://www.dolcn.com 